\title{
Condutividade hidráulica, porosidade, resistência mecânica e intervalo hídrico ótimo em Latossolos artificialmente compactados
}

\author{
Euriel Millan R. ${ }^{1}$, Hugo A. Ruiz ${ }^{2}$, Raphael B. A. Fernandes ${ }^{3} \&$ Liovando M. da Costa $^{4}$ \\ ${ }^{1}$ Faculdade de Engenharia, Universidade de Sucre, Colômbia. E-mail: euriel.millan@unisucre.edu.co \\ ${ }^{2}$ CCA/UFES, Alegre, ES. E-mail: hruiz@ufv.br. Bolsista CAPES e CNPq \\ ${ }^{3}$ DPS/UFV, Viçosa, MG. E-mail: raphael@ufv.br (Autor correspondente) \\ ${ }^{4}$ DPS/UFV, Viçosa, MG. E-mail: liovandomc@yahoo.com.br
}

\section{Palavras-chave:}

índice de compactação

ensaio de compactação de Proctor

densidade do solo

potencial mátrico

\begin{abstract}
R E S U M O
A quantificação do impacto do uso e do manejo na compactação do solo, é fundamental no desenvolvimento de sistemas agrícolas sustentáveis. Objetivou-se, no trabalho realizado com amostras de dois Latossolos argilosos, quantificar a condutividade hidráulica em meio saturado, a macro e a microporosidade em resposta ao índice de compactação definido pela relação entre a densidade do solo e a densidade máxima determinada pelo ensaio de compactação de Proctor, além de quantificar a resistência à penetração em resposta ao indice de compactação no intervalo de -60 a $-15.000 \mathrm{hPa}$ e calcular o intervalo hídrico ótimo. As unidades experimentais foram cilindros de solo artificialmente compactados em laboratório para atingir índices de compactação no intervalo de 0,70 a 1,0. Os resultados mostraram a importância do uso desse índice na comparação de características de solos em que os valores absolutos da sua densidade apresentam acentuada divergência. $O$ índice de compactação na faixa de 0,70 a 0,85 não oferece restrições ao crescimento das plantas. Valores superiores indicam, inicialmente, restrições à aeração do solo e, quando excedem 0,95 , impedimento ao crescimento de raízes pela resistência à penetração.
\end{abstract}

Key words: compaction index Proctor compaction test bulk density matric potential

\section{Hydraulic conductivity, porosity, mechanical resistance and least limiting water range in artificially compacted Oxisols}

\begin{abstract}
A B S T R A C T
The evaluation of the impact of use and management on soil compaction is critical in the development of sustainable agricultural systems. The objective of this study was to conduct with two samples of two clayey Oxisols, quantification of the saturated hydraulic conductivity, the macro and the micro porosity in response to compaction index, defined by the relationship between soil bulk density and maximum density determined by Proctor compaction test. The penetration resistance in response to compaction index between -60 and $-15,000 \mathrm{hPa}$ was also quantified, and calculated the least limiting water range. The experimental units were artificially compacted soil cores in laboratory, in order to achieve compaction indexes between 0.70 and 1.0. The results showed the importance of this index when comparing characteristics of soil in which the absolute values of bulk density exhibit marked divergence. The compaction index between 0.70 and 0.85 offers no restrictions to plant growth. Higher values indicate restrictions with respect to soil aeration, and when the values exceed 0.95 , the penetration resistance impedes root growth.
\end{abstract}

\section{INTRODUÇÃO}

Associada ao crescimento das plantas, a qualidade física do solo é determinada fundamentalmente pela disponibilidade de água e ar e pela resistência que a matriz do solo oferece ao crescimento das raízes. Alterações significativas que ocorram na estrutura do solo em resposta à compactação, entre outras, provocarão mudanças na relação solo-água-ar e na resistência mecânica e, em consequência, no crescimento dos vegetais em resposta às características físicas do solo (Ahmad et al., 2009; Fernández et al., 2010; Kim et al., 2010; Weisskopf et al., 2010).

Estudos relativos ao processo de compactação têm utilizado ensaios frequentemente desenvolvidos para uso em mecânica de solos (Lipiec \& Hatano, 2003; Duruoha et al., 2008; Reichert et al., 2009; Saffih-Hdadi et al., 2009). Exemplo disto é a aplicação do ensaio de compactação de Proctor (ECP) ainda pouco utilizado em estudos de solos tropicais com fins agrícolas. Com dados do ECP, Barber et al. (1989) propuseram a utilização do índice de compactação (IC) calculado pela relação entre a densidade do solo $\left(\mathrm{D}_{\mathrm{S}}\right)$ e a densidade máxima determinada no $\operatorname{ECP}\left(\mathrm{D}_{\mathrm{MP}}\right)$.

O penetrômetro é instrumento utilizado para avaliar o estado de estrutura do solo e a eventual restrição ao crescimento das raízes. Suas medidas são afetadas pelo conteúdo de água, pela composição granulométrica do solo e sua mineralogia (Moraes et al., 2014). Têm sido propostos valores críticos de 
impedância mecânica para diferentes solos e plantas, geralmente na faixa de 1 a $3 \mathrm{MPa}$ (Taylor et al., 1966; Gerard et al., 1982; Pérez \& Diaz-Fierros, 1989).

Outro indicador utilizado para a avaliação da qualidade física do solo é o Intervalo de Água Não Limitante (Non-limiting Water Range), proposto por Letey (1985). Esta característica foi denominada Intervalo Hídrico Ótimo (IHO) (Least Limiting Water Range) e sua aplicação aprofundada por Silva et al. (1994). Esses autores a definiram como a faixa de conteúdo de água associada com o potencial matricial, a aeração e a resistência mecânica, em que ocorrem limitações físicas mínimas para o crescimento das plantas. A vantagem fundamental da utilização do IHO para avaliar a qualidade física do solo é a integração, em única representação, de características físicas diretamente relacionadas ao crescimento das plantas.

O objetivo deste trabalho, realizado com amostras de um Latossolo Vermelho argiloso e de um Latossolo VermelhoAmarelo muito argiloso, foi quantificar, em laboratório, a condutividade hidráulica em meio saturado, a macro e a microporosidade em resposta ao incremento artificial do IC; determinar a resistência à penetração $(\mathrm{RP})$ em relação ao $\mathrm{IC}$ e ao potencial de água do solo e delimitar o IHO.

\section{Material e Métodos}

Foram utilizadas amostras de um Latossolo Vermelho (LV), do município de Capinópolis, MG, e um Latossolo VermelhoAmarelo (LVA), do município de Viçosa, MG. As amostras foram retiradas nas profundidades de 40 a $60 \mathrm{~cm}$ e, na sequência, secadas ao ar, passadas em peneira de $2 \mathrm{~mm}$ e caracterizadas fisicamente (Tabela 1). A caracterização mineralógica da fração argila evidenciou predomínio de caulinita e proporções menores de gibbsita, goethita e hematita.

Tabela 1. Caracterização física das amostras dos Latossolos* peneiradas por malha de $2 \mathrm{~mm}$

\begin{tabular}{|c|c|c|}
\hline Característica & LV & LVA \\
\hline Areia grossa $\left(\mathrm{g} \mathrm{kg}^{-1}\right)^{1 /}$ & 130 & 140 \\
\hline Areia fina $\left(\mathrm{g} \mathrm{kg}^{-1}\right)^{1 /}$ & 120 & 60 \\
\hline Silte $\left(\mathrm{g} \mathrm{kg}^{-1}\right)^{1 /}$ & 160 & 60 \\
\hline Argila $\left(\mathrm{g} \mathrm{kg}^{-1}\right)^{1 /}$ & 590 & 740 \\
\hline Densidade do solo (Método da proveta) $\left(\mathrm{kg} \mathrm{dm}^{-3}\right)^{2 /}$ & 1,160 & 0,970 \\
\hline Densidade de partículas $\left(\mathrm{kg} \mathrm{dm}^{-3}\right)^{2 /}$ & 2,830 & 2,660 \\
\hline Porosidade $\left(\mathrm{m}^{3} \mathrm{~m}^{-3}\right)^{2 /}$ & 0,590 & 0,635 \\
\hline Macroporosidade $\left(\mathrm{m}^{3} \mathrm{~m}^{-3}\right)^{2 /}$ & 0,188 & 0,209 \\
\hline Microporosidade $\left(\mathrm{m}^{3} \mathrm{~m}^{-3}\right)^{2 /}$ & 0,402 & 0,426 \\
\hline Densidade máxima de Proctor $\left(\mathrm{kg} \mathrm{dm}^{-3}\right)^{3 /}$ & 1,640 & 1,370 \\
\hline Porosidade na densidade máxima de Proctor $\left(\mathrm{m}^{3} \mathrm{~m}^{-3}\right)^{3 /}$ & 0,421 & 0,485 \\
\hline Umidade crítica de compactação base gravimétrica $\left(\mathrm{kg} \mathrm{kg}^{-1}\right)^{3 /}$ & 0,244 & 0,324 \\
\hline $\begin{array}{l}\text { Umidade crítica de compactação base volumétrica }\left(\mathrm{m}^{3} \mathrm{~m}^{-3}\right)^{3 /} \\
\text { Retencão de áqua }\left(\mathrm{kg} \mathrm{kq}^{-1}\right)^{4 /}\end{array}$ & 0,400 & 0,444 \\
\hline$-60 \mathrm{hPa}$ & 0,346 & 0,417 \\
\hline$-100 \mathrm{hPa}$ & 0,276 & 0,359 \\
\hline$-300 \mathrm{hPa}$ & 0,206 & 0,278 \\
\hline$-1.000 \mathrm{hPa}$ & 0,186 & 0,256 \\
\hline$-5.000 \mathrm{hPa}$ & 0,176 & 0,249 \\
\hline$-15.000 \mathrm{hPa}$ & 0,170 & 0,235 \\
\hline
\end{tabular}

As unidades experimentais utilizadas no ensaio foram tubos cilíndricos de aço inoxidável, de 4,8 cm de diâmetro e 5,0 cm de altura, preenchidos com amostra de solo até $3,0 \mathrm{~cm}$. Em todos os ensaios foram testados quatro IC: 0,7, 0,8, 0,9 e 1,0. Quantidades de amostras peneiradas em malha de $2 \mathrm{~mm}$, previamente calculadas para formar cilindros de $4,8 \mathrm{~cm}$ de diâmetro e 3,0 $\mathrm{cm}$ de altura nos IC estabelecidos, receberam volume de água correspondente à umidade crítica de compactação (Tabela 1) e foram compactadas através de prensa hidráulica.

A condutividade hidráulica em meio saturado foi determinada segundo um arranjo fatorial de dois solos (LV e LVA) e quatro IC $(0,7 ; 0,8 ; 0,9$ e 1,0$)$, num delineamento experimental inteiramente casualizado com quatro repetições.

As unidades experimentais, preparadas da forma previamente indicada, foram saturadas por $48 \mathrm{~h}$. Seguidamente, foram colocadas em permeâmetros de carga constante para determinar a condutividade hidráulica em meio saturado (Freire et al., 2003).

Finalizado o ensaio, a unidade experimental foi imediatamente transferida à mesa de tensão para determinar a microporosidade, por equilíbrio a tensão de $60 \mathrm{~cm}$ de coluna de água, por 48 h (EMBRAPA, 1997). A umidade base gravimétrica da amostra multiplicada pela $\mathrm{D}_{\mathrm{s}}$ correspondente a cada IC de trabalho permitiu calcular a umidade base volumétrica, valor correspondente à microporosidade. A porosidade total foi calculada subtraindo-se, da unidade, a relação entre $a D_{s}$ e a densidade de partículas e a macroporosidade, subtraindo-se a microporosidade da porosidade total.

Os resultados experimentais foram analisados estatisticamente comparando-se os solos pelo teste $\mathrm{F}$ e a resposta ao IC, para cada solo, por meio de equações de regressão.

A RP foi determinada segundo um arranjo fatorial de dois solos (LV e LVA), quatro IC $(0,7 ; 0,8 ; 0,9$ e 1,0$)$ e cinco potenciais matriciais $(-60,-100,-300,-1.000$ e $-15.000 \mathrm{hPa})$, num delineamento experimental inteiramente casualizado com três repetições.

As unidades experimentais, preparadas da forma previamente indicada, foram saturadas por $48 \mathrm{~h}$. Seguidamente, as correspondentes aos tratamentos de -60 e $-100 \mathrm{hPa}$ foram colocadas em mesa de tensão e as de $-300,-1.000 \mathrm{e}-15.000 \mathrm{hPa}$, em equipamento de placa de pressão. Após $72 \mathrm{~h}$ determinou-se a RP por meio de um penetrômetro de bancada. Os gráficos gerados permitiram determinar a RP em toda a profundidade do cilindro de solo $(3 \mathrm{~cm})$. Os dados utilizados correspondem à $\mathrm{RP}$ determinada $1,5 \mathrm{~cm}$ de profundidade.

Os resultados experimentais foram analisados estatisticamente comparando-se os solos pelo teste F e a resposta ao IC e ao potencial, para cada solo, por meio de superfícies de resposta.

Quatro curvas foram representadas para determinar o IHO utilizando-se a $\mathrm{D}_{\mathrm{S}}$, calculada multiplicando-se o IC pela $\mathrm{D}_{\mathrm{MP}}$ (Tabela 1) como variável independente. As variáveis dependentes foram a porosidade de aeração superior a $0,10 \mathrm{~m}^{3} \mathrm{~m}^{-3}\left(\mathrm{P}_{\mathrm{A}>10}\right) \mathrm{e}$ os conteúdos de água base volumétrica determinados a -100 $\mathrm{hPa}\left(\theta_{\mathrm{CC}}\right), \mathrm{a}-15.000 \mathrm{hPa}\left(\theta_{\mathrm{PMP}}\right)$ e aquele em que a resistência mecânica do solo é de 1,0 MPa $\left(\theta_{\mathrm{RP}}\right)$.

A $P_{A>10}$ foi calculada com base na $D_{M P}$ e na densidade de partículas $\left(\mathrm{D}_{\mathrm{p}}\right)$, para os diferentes IC, pela Eq. 1 . 


$$
\mathrm{P}_{\mathrm{A}>10}=\left(1-\frac{\mathrm{D}_{\mathrm{MP}} \mathrm{IC}}{\mathrm{D}_{\mathrm{P}}}\right)-0,10
$$

O conteúdo de água base volumétrica $(\theta)$ foi calculado a partir do conteúdo de água base gravimétrica (U), pela Eq. 2.

$$
\theta=U \frac{\mathrm{D}_{\mathrm{MP}} \mathrm{IC}}{\mathrm{D}_{\text {Agua }}}
$$

O IHO foi delimitado na porção superior, pelas equações calculadas para $\theta_{\mathrm{CC}}$ e para $\mathrm{P}_{\mathrm{A}>10}$ e na porção inferior pelas equações calculadas para $\theta_{\mathrm{PMP}}$ e para $\theta_{\mathrm{RP}}$. A superfície entre o menor valor da porção superior e o maior valor da porção inferior delimitou o IHO.

\section{Resultados e Discussão}

O menor IC utilizado (0,70), quando multiplicado pelas respectivas $D_{M P}$ (Tabela 1), resultou em valores de 1,15 e 0,96 $\mathrm{kg} \mathrm{dm}^{-3}$ para o LV e o LVA, respectivamente. Na comparação com aqueles apresentados na caracterização, determinados pelo método da proveta, observam-se valores de 1,16 e 0,97 $\mathrm{kg} \mathrm{dm}^{-3}$ (Tabela 1) sugerindo que em ensaios de compactação de solos valores de IC próximos a 0,70 seriam utilizados apropriadamente como representativos de condições próximas às normalmente encontradas em Latossolos não cultivados.

Os valores da condutividade hidráulica em meio saturado $\left(\mathrm{K}_{0}\right)$, da macroporosidade $\left(\mathrm{P}_{\mathrm{M}}\right)$ e da microporosidade $\left(\mathrm{P}_{\mathrm{m}}\right)$, determinados em resposta ao IC, para os dois solos estudados (LV e LVA), são apresentados na Tabela 2. Diferenças estatísticas significativas na comparação dos solos podem ser atribuídas, fundamentalmente, aos baixos valores dos coeficientes de variação: $3,74,1,75$ e 0,60\% para as três determinações, respectivamente.

A $\mathrm{K}_{0}$ decresceu acentuadamente com o IC nos dois solos estudados (Figura 1). Como o movimento de água em meio saturado se realiza através dos grandes poros (Soracco et al., 2012), a diminuição de $K_{0}$ deveria associar-se com tendência semelhante na $\mathrm{P}_{\mathrm{M}}$, como mostrado também na Figura 1. Quando relacionada $\mathrm{K}_{0}$ com $\mathrm{P}_{\mathrm{M}}$ e $\mathrm{P}_{\mathrm{m}}$, os coeficientes de correlação linear simples foram de $0,978^{\star *}$ e $-0,351^{\text {ns }}$, respectivamente.

Tabela 2. Condutividade hidráulica em meio saturado $\left(\mathrm{K}_{0}\right)$, macroporosidade $\left(\mathrm{P}_{\mathrm{M}}\right)$ e microporosidade $\left(\mathrm{P}_{\mathrm{m}}\right)$, considerando-se o solo e o índice de compactação

\begin{tabular}{|c|c|c|c|c|c|c|}
\hline \multirow{2}{*}{ IC } & \multicolumn{3}{|c|}{$\mathbf{L V}^{*}$} & \multicolumn{3}{|c|}{ LVA $^{*}$} \\
\hline & $\mathrm{K}_{0}$ & $\mathbf{P}_{\mathrm{M}}$ & $\mathbf{P}_{\mathrm{m}}$ & $\mathrm{K}_{0}$ & $\mathbf{P}_{\mathrm{M}}$ & $\mathbf{P}_{\mathrm{m}}$ \\
\hline & $\mathrm{cm} \mathrm{h}^{-1}$ & \multicolumn{2}{|c|}{$\mathrm{m}^{3} \mathrm{~m}^{-3}$} & $\mathrm{~cm} \mathrm{~h}^{-1}$ & \multicolumn{2}{|c|}{$\mathrm{m}^{3} \mathrm{~m}^{-3}$} \\
\hline 0,70 & 13,90 & 0,224 & 0,370 & 14,20 & 0,235 & 0,404 \\
\hline 0,80 & 11,18 & 0,159 & 0,375 & 11,83 & 0,174 & 0,413 \\
\hline 0,90 & 4,13 & 0,097 & 0,380 & 4,43 & 0,119 & 0,418 \\
\hline 1,00 & 0,40 & 0,035 & 0,385 & 0,53 & 0,048 & 0,437 \\
\hline Média ${ }^{1 /}$ & $7,40 \mathrm{~B}$ & $0,129 \mathrm{~B}$ & $0,377 \mathrm{~B}$ & $7,74 \mathrm{~A}$ & $0,144 \mathrm{~A}$ & $0,418 \mathrm{~A}$ \\
\hline
\end{tabular}
(IC) estudados

* LV - Latossolo Vermelho; LVA - Latossolo Vermelho Amarelo

${ }^{1 /}$ Letras diferentes na linha, para a mesma característica, indicam diferenças estatisticamente significativas a 0,01 pelo teste $\mathrm{F}$
Da análise das duas equações de regressão para $\mathrm{P}_{\mathrm{M}}$ e $\mathrm{P}_{\mathrm{m}}$ (Figura 1), verifica-se que $\mathrm{P}_{\mathrm{M}}$ apresenta declividade com sinal negativo sendo positiva para $\mathrm{P}_{\mathrm{m}}$. Por outra parte, relacionando os módulos das declividades de $\mathrm{P}_{\mathrm{M}}$ e $\mathrm{P}_{\mathrm{m}}\left(\mathrm{P}_{\mathrm{M}} / \mathrm{P}_{\mathrm{m}}\right)$, os valores calculados são de 12,3 e 5,9 para LV e LVA, respectivamente, o que indica que a diminuição da $\mathrm{P}_{\mathrm{M}}$ não é acompanhada por

A.

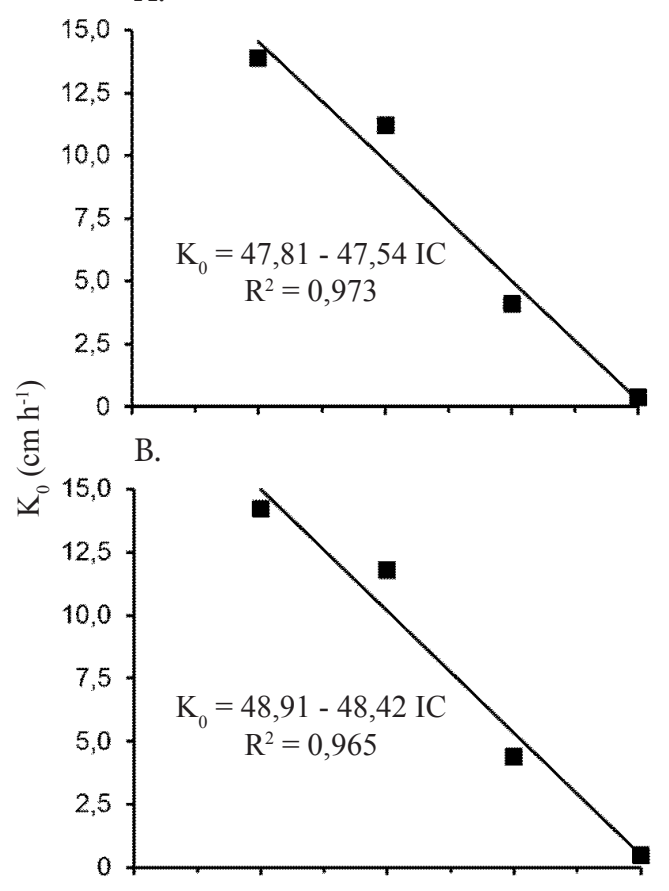

C.

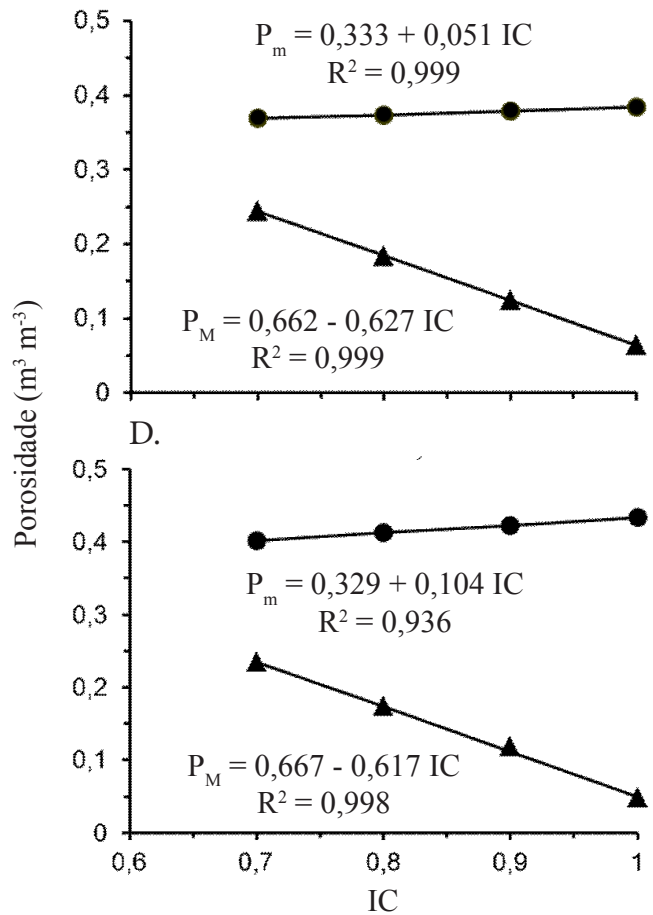

Os coeficientes das equações de regressão são significativos a 0,01 pelo teste t

Figura 1. Condutividade hidráulica em meio saturado $\left(\mathrm{K}_{0}\right)$ (A e B), macroporosidade $\left(\mathrm{P}_{\mathrm{M}}\right)$ e microporosidade $\left(P_{m}\right)(C$ e D) em função do índice de compactação (IC), em amostras dos solos estudados (A e C: Latossolo Vermelho e B e D: Latossolo Vermelho Amarelo) 
incremento equivalente da $\mathrm{P}_{\mathrm{m}}$. $\mathrm{O}$ resultado é a diminuição acentuada da porosidade total.

Considerando a RP, os dois solos apresentaram valores extremos de 0,061 e 4,217 MPa para o LV e de 0,114 e 2,028 $\mathrm{MPa}$, para o LVA. Quando comparados os solos, a RP no LV foi estatisticamente maior que no LVA, com médias gerais de $1,01 \mathrm{e}$ 0,702 MPa, respectivamente. Como os tratamentos levaram em consideração o potencial e não o conteúdo de água do solo, a diferença na retenção de água nos potenciais de trabalho (Tabela 1) justifica os valores diferenciados.

A resposta da $\mathrm{RP}$ ao IC e à energia de retenção de água para cada solo, foi analisada por meio de superfícies de resposta. Utilizou-se a tensão $(\mathrm{T})$ e não o potencial $(\Psi)$ para facilitar o ajuste das equações correspondentes considerando-se que $\mathrm{T}=-\Psi$. Utilizou-se, também, como variável independente, $\mathrm{o}$ conteúdo de água base gravimétrica associado a cada potencial de trabalho (U) (Tabela 3).

Para facilitar a visualização das respostas da RP ao IC e a $\mathrm{T}$ (ou U), as superfícies de resposta apresentadas na Tabela 3 sofreram cortes nos valores correspondentes a cada IC de trabalho $(0,70 ; 0,80 ; 0,90$ e 1,0$)$. As curvas resultantes são apresentadas na Figura 2.

Considerando que a tensão apresenta sinal oposto ao potencial da água do solo, divergentes também são as curvas de resposta da RP, com tendência ao crescimento quando representadas em função da tensão e decrescentes quando utilizado o conteúdo de água como variável independente (Figura 2). Pela análise das figuras verifica-se, de imediato, a resposta diferenciada dos dois solos aos IC testados. O LV apresenta diferenças mais acentuadas que o LVA e atinge valores superiores de RP até superar $3 \mathrm{MPa}$. Essa resposta é coerente com os valores da $\mathrm{D}_{\mathrm{MP}}$, determinada na caracterização. $\mathrm{O} \mathrm{LV}$ e o LVA tiveram valores de 1,64 e 1,37 $\mathrm{kg} \mathrm{dm}^{-3}$, respectivamente (Tabela 1).

As diferenças entre solos são mais bem observadas na Figura 2 quando a RP é representada em função de U. Em todos os potenciais de trabalho a umidade em equilíbrio do material mais argiloso (LVA) foi superior à do LV (Tabela 1), justificando a menor diferença entre o IC e os menores valores da RP. Em resposta ao incremento da umidade do solo ocorre variação, em sentido inverso, na RP, em resposta à ação da água na redução da coesão das partículas do solo.

Do ponto de vista físico, as variações no conteúdo de água no solo provocam modificações na consistência, pelo grau de coesão da massa de solo e da adesão da água aos constituintes da fase sólida. Assim, os solos são mais resistentes às pressões na baixa umidade do solo e mais susceptíveis à compactação, com elevada umidade do solo (Horn et al., 1995).
A.

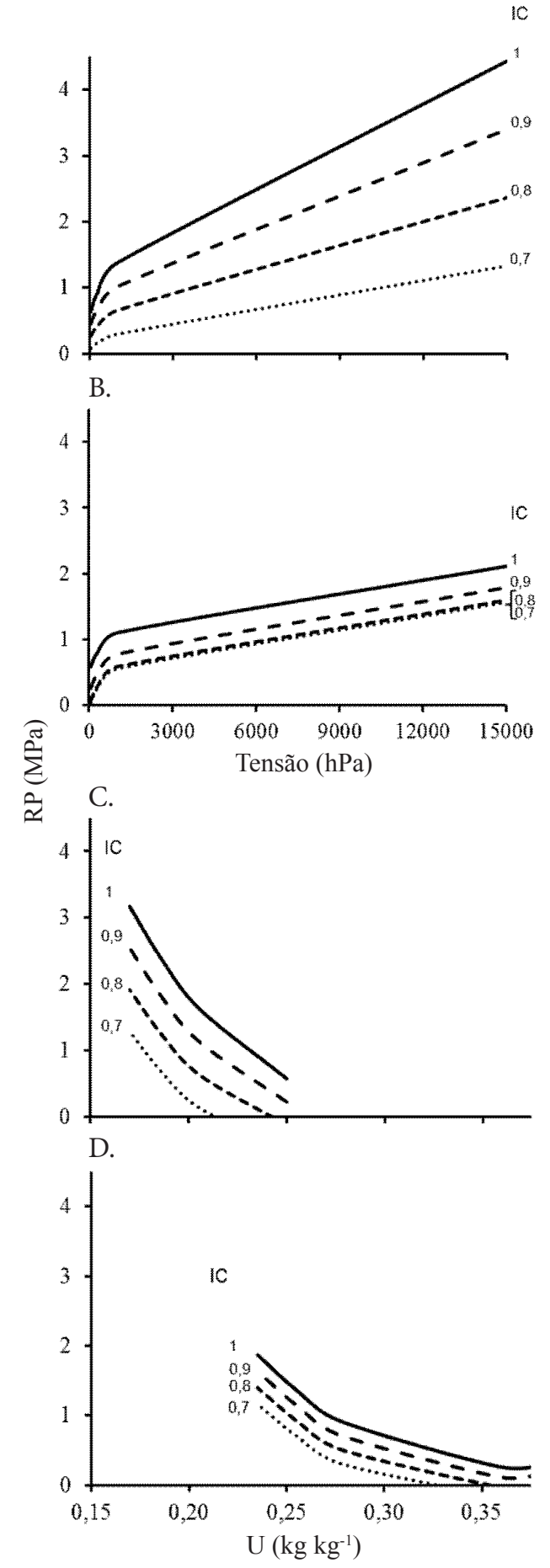

Figura 2. Resistência à penetração (RP) em resposta à tensão da água do solo (A e B) e ao conteúdo de água do solo (U) (C e D) sob diferentes índices de compactação (IC), em amostras dos solos estudados: Latossolo Vermelho (A e C) e Latossolo Vermelho Amarelo (C e D)

Tabela 3. Superfícies de resposta relacionando-se a resistência à penetração (RP) a 1,5 cm de profundidade com o índice de compactação (IC) e a tensão (T) ou o conteúdo de água do solo, base gravimétrica (U)

\begin{tabular}{llll}
\hline \multicolumn{1}{c}{ Solo } & \multicolumn{1}{c}{ Superfície de resposta } & $\mathbf{R}^{2}$ \\
Latossolo Vermelho & $\mathrm{RP}=-0,853+1,249 \mathrm{IC}+0,0000220 \mathrm{~T}-0,0440 \mathrm{~T}^{0,5}+0,0743 \mathrm{IC} \mathrm{T}^{0,5}$ & 0,988 \\
Latossolo Vermelho Amarelo & $\mathrm{RP}=19,135+26,397 \mathrm{IC}-45,136 \mathrm{IC} \mathrm{C}^{0,5}-0,0000914 \mathrm{~T}+0,0252 \mathrm{~T}^{0,5}$ & 0,970 \\
Latossolo Vermelho & $\mathrm{RP}=31,085+19,359 \mathrm{IC}+170,135 \mathrm{U}-153,085 \mathrm{U}^{0,5}-31,725 \mathrm{IC} \mathrm{U}^{0,5}$ & 0,722 \\
Latossolo Vermelho Amarelo & $\mathrm{RP}=36,754+6,089 \mathrm{IC}+117,655 \mathrm{U}-133,851 \mathrm{U}^{0,5}-7,706 \mathrm{IC} \mathrm{U}^{0,5}$ & 0,840 \\
\hline
\end{tabular}


$\mathrm{Na}$ Tabela 4 são apresentados os dados da $\mathrm{D}_{\mathrm{s}}$, da porosidade de aeração superior a $0,10 \mathrm{~m}^{3} \mathrm{~m}^{-3}\left(\mathrm{P}_{\mathrm{A}>10}\right)$, do conteúdo de água base volumétrica a $-100 \mathrm{hPa}$ e $-15.000 \mathrm{hPa}\left(\theta_{\mathrm{CC}}\right.$ e $\left.\theta_{\mathrm{PMP}}\right)$ e do conteúdo de água determinado para resistência mecânica do solo de 1,0 MPa $\left(\theta_{\mathrm{RP}}\right)$, considerando-se o IC, para os dois solos estudados.

O conteúdo de água base volumétrica que permite manter a RP num valor prefixado de 1,0 MPa, quarta variável a ser levada em consideração para a determinação do IHO, foi calculado a partir das superfícies de resposta indicadas na Tabela 3. Utilizando as funções $\mathrm{RP}=\mathrm{f}(\mathrm{IC}, \mathrm{U})$, determinou-se o conteúdo de água base gravimétrica $(\mathrm{U})$ necessário para permitir RP de 1,0 $\mathrm{MPa}$, nos IC de trabalho. $\theta_{\mathrm{RP}}$ foi calculado utilizando-se a Eq. 2 .

Os valores apresentados na Tabela 4 foram utilizados para ajustar equações de regressão utilizadas para delimitar o IHO nos solos estudados (Tabela 5).

As equações que representaram $P_{A>10}=f\left(D_{S}\right)$ e $\theta_{R P}=f\left(D_{S}\right)$ (Tabela 5) foram utilizadas para determinar a densidade do solo crítica $\left(\mathrm{D}_{\mathrm{sc}}\right)$, definida como aquela que limita o IHO. Sendo este ponto a $D_{S}$ que permite a interseção entre valores das duas equações indicadas, realiza-se o cálculo de $\mathrm{D}_{\mathrm{SC}}$ igualando $P_{A>10}$ e $\theta_{R P}$. Os valores assim calculados foram 1,62 e $1,38 \mathrm{~kg} \mathrm{dm}^{-3}$ para o LV e LVA, respectivamente. Considerando a

Tabela 4. Densidade do solo $\left(D_{\mathrm{s}}\right)$, porosidade de aeração superior a $0,10 \mathrm{~m}^{3} \mathrm{~m}^{-3}\left(\mathrm{P}_{\mathrm{A}>10}\right)$, conteúdo de água base volumétrica a $-100 \mathrm{hPa}$ e $-15.000 \mathrm{hPa}\left(\theta_{\mathrm{CC}} \mathrm{e}\right.$ $\left.\theta_{\mathrm{PMP}}\right)$ e conteúdo de água determinado para resistência mecânica do solo de 1,0 MPa $\left(\theta_{\mathrm{RP}}\right)$ considerando-se o índice de compactação (IC)

\begin{tabular}{|c|c|c|c|c|c|c|}
\hline \multirow{2}{*}{ Solo* } & \multirow{2}{*}{ IC } & \multirow{2}{*}{$\begin{array}{c}D_{s} \\
\mathrm{~kg} \mathrm{dm}^{-3}\end{array}$} & $P_{A>10}$ & $\theta_{c c}$ & $\theta_{\text {PMP }}$ & $\theta_{\mathrm{RP}}$ \\
\hline & & & \multicolumn{4}{|c|}{$\mathbf{m}^{3} \mathbf{m}^{-3}$} \\
\hline \multirow{4}{*}{ LV } & 0,70 & 1,148 & 0,494 & 0,317 & 0,195 & 0,197 \\
\hline & 0,80 & 1,312 & 0,436 & 0,362 & 0,223 & 0,241 \\
\hline & 0,90 & 1,476 & 0,378 & 0,407 & 0,251 & 0,275 \\
\hline & 1,00 & 1,640 & 0,320 & 0,453 & 0,279 & 0,376 \\
\hline \multirow{4}{*}{ LVA } & 0,70 & 0,959 & 0,539 & 0,344 & 0,225 & 0,235 \\
\hline & 0,80 & 1,096 & 0,488 & 0,393 & 0,258 & 0,269 \\
\hline & 0,90 & 1,233 & 0,436 & 0,443 & 0,290 & 0,313 \\
\hline & 1,00 & 1,370 & 0,385 & 0,492 & 0,322 & 0,370 \\
\hline
\end{tabular}

* LV - Latossolo Vermelho; LVA - Latossolo Vermelho Amarelo

Tabela 5. Equações de regressão relacionando-se a porosidade de aeração superior a $0,10 \mathrm{~m}^{3} \mathrm{~m}^{-3}$ $\left(\mathrm{P}_{\mathrm{A}>10}\right)$, o conteúdo de água base volumétrica a -100 hPa e $-15.000 \mathrm{hPa}\left(\theta_{C \mathrm{C}}\right.$ e $\left.\theta_{\mathrm{PMP}}\right)$ e o conteúdo de água determinado para resistência mecânica do solo de 1,0 MPa $\left(\theta_{\mathrm{RP}}\right)$ com a densidade do solo $\left(\mathrm{D}_{\mathrm{s}}\right)$

\begin{tabular}{lc}
\hline \multicolumn{1}{c}{ Equação de regressão } & $\mathbf{R}^{2}$ \\
$\mathrm{P}_{\mathrm{A}>10}=0,900-0,353 \mathrm{D}_{\mathrm{S}}$ & \\
$\theta_{\mathrm{CC}}=0,276 \mathrm{D}_{\mathrm{S}}$ & 0,999 \\
$\theta_{\mathrm{PMP}}=0,170 \mathrm{D}_{\mathrm{S}}$ & 0,999 \\
$\theta_{\mathrm{RP}}=1 /\left(10,427-4,698 \mathrm{D}_{\mathrm{S}}\right)$ & 0,999 \\
\multicolumn{2}{c}{ Latossolo Vermelho Amarelo } \\
$\mathrm{P}_{\mathrm{A}>10}=0,894-0,372 \mathrm{D}_{\mathrm{S}}$ & 0,972 \\
$\theta_{\mathrm{CC}}=0,359 \mathrm{D}_{\mathrm{S}}$ & 0,999 \\
$\theta_{\mathrm{PMP}}=0,235 \mathrm{D}_{\mathrm{S}}$ & 0,999 \\
$\theta_{\mathrm{RP}}=1 /\left(7,880-3,788 \mathrm{D}_{\mathrm{S}}\right)$ & 0,999 \\
\hline & 0,999 \\
\hline
\end{tabular}

$\mathrm{D}_{\mathrm{MP}}$ (Tabela 1), esses valores correspondem a IC de 0,99 e 1,01 para o LV e LVA, respectivamente; entretanto, efeitos negativos sobre o desenvolvimento vegetal podem ocorrer bem antes de se alcançar a $\mathrm{D}_{\mathrm{SC}}$ Klein (2006) indica um efeito limitante a partir de IC de 0,88 para um Latossolo Vermelho argiloso e Beutler et al. (2005) perceberam redução de ganhos na cultura de soja a partir dos máximos alcançados de produtividade associados aos valores de IC de 0,75 e 0,84, em dois Latossolos Vermelhos de diferentes mineralogias. Já Reichert et al. (2009) verificaram redução de produção a partir de valores de IC calculados com o ensaio de Proctor de 0,90 e 0,95 para duas diferentes culturas.

A Figura 3 mostra a representação gráfica do $\mathrm{IHO}$ calculado com as curvas ajustadas que foram apresentadas no quadro 5 , e a $\mathrm{D}_{\mathrm{SC}}$. Como se pode observar, o IHO é limitado na porção superior da área pela disponibilidade de água na capacidade de campo ou pela restrição à aeração do sistema radicular. $\mathrm{Na}$ porção inferior as limitações se devem à RP.

Na porção superior do IHO a disponibilidade de água na capacidade de campo é limitante nas menores $\mathrm{D}_{\mathrm{s}}$. Assim que a compactação é incrementada a restrição à aeração delimita o IHO (Figura 3). Comparando as curvas que representam $P_{A>10}$ e $\theta_{C C}$ em função da $D_{S}$ (Tabela 5), determinam-se valores de $\mathrm{D}_{\mathrm{S}}$ de 1,43 e $1,22 \mathrm{~kg} \mathrm{dm}^{-3}$ para o LV e LVA, respectivamente, para a transição apontada. Esses valores, claramente discrepantes, se transformam em IC de 0,87 e 0,89, quando divididos pela $\mathrm{D}_{\mathrm{MP}}$ (Tabela 1). A convergência de resultados é indicativa da importância do uso de valores relativos na comparação de características de solos em que os valores absolutos apresentam acentuada divergência. Os resultados de IC obtidos também extrapolam aqueles indicados por Klein (2006) e Beutler et al. (2005) para Latossolos brasileiros, passíveis de sugerir que efeitos no desenvolvimento vegetal podem ocorrer antes que a porosidade de aeração de $0,10 \mathrm{~m}^{3} \mathrm{~m}^{-3}$ seja alcançada.

Na porção inferior do IHO o conteúdo de água determinado para a RP de 1,0 MPa foi limitante em relação ao conteúdo de água determinado no ponto de murcha permanente (Figura 3 ).

No IHO se estabelecem limitações à produtividade quando $\mathrm{P}_{\mathrm{A}>10}$ diminui a valores inferiores a $0,10 \mathrm{~m}^{3} \mathrm{~m}^{-3}$. Este limite deveria ser revisado para adequá-lo às diferentes espécies vegetais e, ainda, aos variados materiais genéticos dentro de cada espécie, com o que concordam Reichert et al. (2009). De forma semelhante trabalha-se, em geral, com RP na faixa de 1,0 a 3,0 MPa. Este limite também exige pesquisas para estabelecer, com maior exatidão, valores condizentes com as características das plantas cultivadas.

Pelos resultados apresentados pode postular-se que a utilização do IC é mais apropriada que o uso de valores de $\mathrm{D}_{\mathrm{s}}$. $\mathrm{O}$ IC inicial de trabalho, de 0,70 , correspondeu aproximadamente ao valor da $D_{s}$ peneirada, determinado pelo método da proveta (Tabela 1). Assim, valores de IC na faixa de 0,70 a 0,85 , aproximadamente, não ofereceriam restrições ao crescimento das plantas. Valores superiores indicariam, de início, restrições à aeração do solo e quando na faixa de 0,95 a 1,0, impedimento ao crescimento de raízes pela RP. 
A.

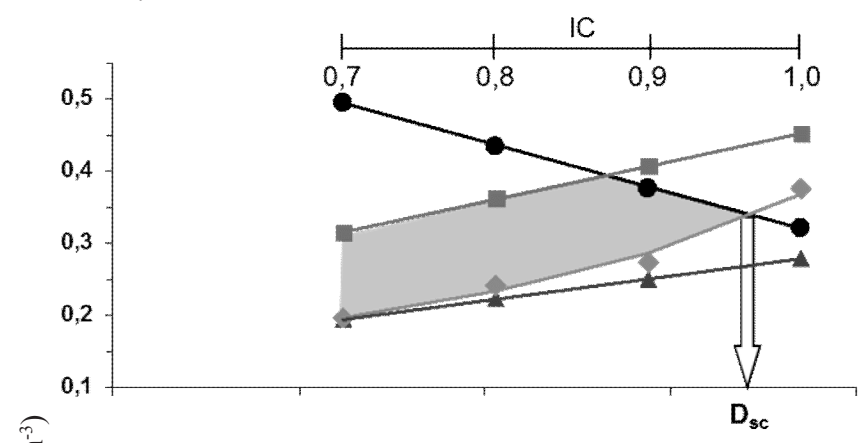

B.

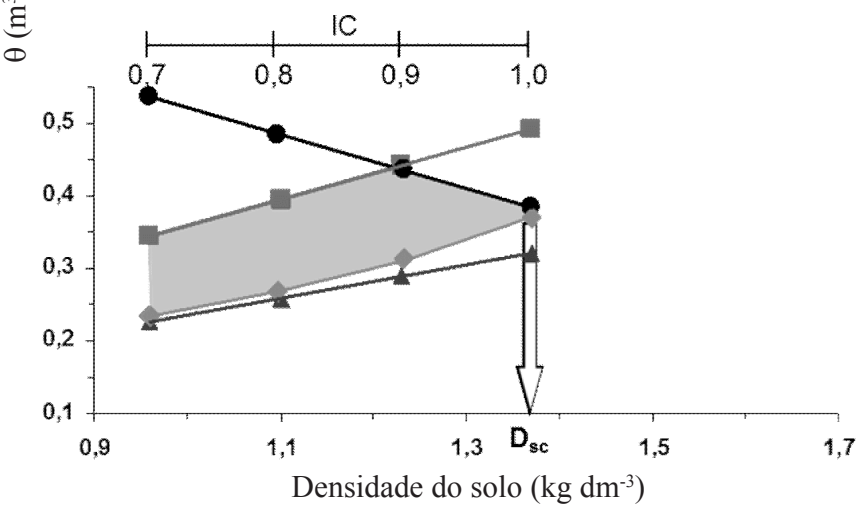

0 IHO é delimitado pela porosidade de aeração superior a $0,10 \mathrm{~m}^{3} \mathrm{~m}^{-3}(\bullet)$ e os conteúdos de água a $-10 \mathrm{kPa}(\mathbf{-})$, a $-1.500 \mathrm{kPa}(\boldsymbol{\Delta})$ e àquele determinado para resistência mecânica do solo de $1 \mathrm{MPa}(\bullet)$

Figura 3. Intervalo hídrico ótimo (IHO) e densidade do solo crítica $\left(\mathrm{D}_{\mathrm{sC}}\right)$, em amostras dos solos estudados: Latossolo Vermelho (A) e Latossolo Vermelho Amarelo (B), considerando-se a densidade do solo $\left(D_{\mathrm{s}}\right)$ e o índice de compactação (IC)

\section{Conclusões}

1. O incremento do índice de compactação reduz a condutividade hidráulica e a macroporosidade, com menor impacto sobre a microporosidade.

2. A resistência à penetração aumenta de forma diferenciada, em função da natureza do solo com o incremento da compactação e a diminuição do potencial da água do solo.

3. A determinação do intervalo hídrico ótimo é complemento adequado para diminuir perdas de produtividade em resposta à compactação.

4. Índice de compactação na faixa de 0,70 a 0,85 não oferece restrições ao crescimento das plantas. Valores superiores indicam, inicialmente, restrições à aeração do solo e quando na faixa de 0,95 a 1,0, impedimento ao crescimento de raízes pela resistência à penetração.

\section{Literatura Citada}

ABNT - Associação Brasileira de Normas Técnicas. Ensaio de compactação - NBR 7182. Rio de Janeiro: Sistema Nacional de Metrologia, Normalização e Qualidade Industrial, 1986. 10p.
Ahmad, N.; Hassan, F. U.; Belford, R. K. Effects of soil compaction in the sub-humid cropping environment in Pakistan on uptake of NPK and grain yield in wheat (Triticum aestivum). Field Crops Research, v.110, p.61-68, 2009.

Barber, R. G.; Herrera, C.; Diaz, O. Compaction status and compaction susceptibility of alluvial soil in Santa Cruz, Bolivia. Soil \& Tillage Research, v.15, p.153-167, 1989.

Beutler, A. N.; Centurion, J. F.; Roque; C. .G.; Ferraz, M. V. Densidade relativa ótima de Latossolos Vermelhos para a produtividade de soja. Revista Brasileira de Ciência do Solo, v.29, p.843-849, 2005.

Duruoha, C.; Piffer, C. R.; Silva, P. R. A. Root volume and dry matter of peanut plants as a function of soil bulk density and soil water stress. Irriga, v.13, p.170-181, 2008.

EMBRAPA - Empresa Brasileira de Pesquisa Agropecuária. Centro Nacional de Pesquisa de Solos. Manual de métodos de análise de solo. 2.ed. Rio de Janeiro: Centro Nacional de Pesquisa de Solos, 1997. 212p.

Fernández, P. L.; Alvarez, C. R.; Schindler, V.; Taboada, M. A. Changes in topsoil bulk density after grazing crop residues under no-till farming. Geoderma, v.159, p.24-30, 2010.

Freire, M. B. G. dos S.; Ruiz, H. A.; Ribeiro, M. R.; Ferreira, P. A.; Alvarez V., V. H.; Freire, F. J. Condutividade hidráulica de solos de Pernambuco em resposta à condutividade elétrica $\mathrm{e}$ RAS da água de irrigação. Revista Brasileira de Engenharia Agrícola e Ambiental, v.7, p.45-52, 2003.

Gerard, C. J.; Sexton, P.; Shaw, G. Physical factors influencing soil strength and root growth. Agronomy Journal, v.74, p.875-879, 1982.

Horn, R.; Domzal, H.; Jurkiewitcz, A.S.; Owerkerk, C. van. Soil compaction processes and their effects on the structure of arable soils and the environment. Soil \& Tillage Research, v.35, p.23-36, 1995.

Kim, H.-M.; Anderson S. H.; Motavalli, P. P.; Gantzer, C. J. Compaction effects on soil macropore geometry and related parameters for an arable field. Geoderma, v.160, p.244-251, 2010.

Klein, V.A. Densidade relativa - um indicador da qualidade física de um latossolo vermelho. Revista de Ciências Agroveterinárias, v.5, p. 26-32, 2006.

Letey, J. Relationship between soil physical properties and crop production. Advances in Soil Science, v.1, p.277-294, 1985.

Lipiec, J.; Hatano, R. Quantification of compaction effects on soil physical properties and crop growth. Geoderma, v.116, p.107-136, 2003.

Moraes, M. T.; Silva, V. R.; Zwirtes, A. L.; Carlesso, R. Use of penetrometers in agriculture: A review. Engenharia Agrícola, v.34, p.179-193, 2014.

Pérez, R.; Díaz-Fierros, F. Resistencias del suelo y susceptibilidad a la compactación en terrenos a monte sometidos a pastoreo. Anales de Edafología y Agrobiología, v.42, p.377-386, 1989.

Reichert, J. M.; Suzuki, L. E. A. S.; Reinert, D. J.; Horn, R.; Haakansson, I. Reference bulk density and critical degreeof-compactness for no-till crop production in subtropical highly weathered soils. Soil \& Tillage Research, v.102, p.242-254, 2009.

Richards, L. A. Methods of measuring soil moisture tension. Soil Science, v.68, p.95-112, 1949. 
Ruiz, H. A. Incremento da exatidão da análise granulométrica do solo por meio da coleta da suspensão (silte + argila). Revista Brasileira de Ciência do Solo, v.29, p.297-300, 2005.

Saffih-Hdadi, K.; Défossez, P.; Richard, G.; Cui, Y.-J.; Tang, A.M.; Chaplain, V. A method for predicting soil susceptibility to the compaction of surface layers as a function of water content and bulk density. Soil \& Tillage Research, v.105, p.96-103, 2009.

Silva, A. P.; Kay, B. D.; Perfect, E. Characterization of the least limiting water range. Soil Science Society of America Journal, v.58, p.1775-1781, 1994.
Soracco, C. G.; Lozano, L. A.; Balbuena, R.; Ressia, J. M.; Filgueira, R. R. Contribution of macroporosity to water flux of a soil under different tillage systems. Revista Brasileira de Ciência do Solo, v.36, p.1149-1156, 2012.

Taylor, H. M.; Roberson, G. M.; Parker Júnior, J. Soil strengthroot penetration relations for medium to coarse textured soil materials. Soil Science, v.102, p.18-22, 1966.

Weisskopf, P.; Reiser, R.; Rek, J.; Oberholzer, H. R. Effect of different compaction impacts and varying subsequent management practices on soil structure, air regime and microbiological parameters. Soil \& Tillage Research, v.111, p.65-74, 2010. 